\title{
A Passive Electrodeless Method for Determining the Interior Field of Biological Materials
}

\author{
MORRIS E. BRODWIN, SENIOR MEMBER, IEEE, ALLEN TAFLOVE, MEMBER, IEEE, \\ AND JOHN E. MATZ, MEMBER, IEEE
}

\begin{abstract}
A passive remote-sensing method is proposed for the measurement of the electric-field distribution within a biological sample. The instrument analyzes the intermodulation spectrum reradiated by a diode imbedded within the sample exposed to a dual-frequency illumination. Experimental results in a waveguide system indicate that the relative intensities of the intermodulation products are a unique function of the field intensity at the diode. These results are also used to determine the basic scattering properties of the diode. A low-power free-space apparatus is described which exposes the principal problem associated with this method: the inadvertent generation of intermodulation frequencies. Isolation techniques are described which eliminate this problem. Finally, an analysis of a complete system shows that at $910 \mathrm{MHz}$ fields can be measured in $4.0 \mathrm{~cm}$ of soft tissue, $\tan \delta=0.5$, without exceeding an incident power density of $10 \mathrm{~mW} / \mathrm{cm}^{2}$.
\end{abstract}

\section{INTRODUCTION}

$\mathrm{O}$ $\mathrm{NE}$ of the recurring problems in the study of the effect of electromagnetic fields upon biological structures is the determination of the fields at any point within the structure [1]. The usual experimental method is to measure the field directly by inserting detecting diodes within the sample and determining the rectified current by externally connected high-resistance leads. The major problem with the directly inserted detecting diode is the effect of the interconnecting leads upon the distribution of the field. In general, there is no positive way of inferring the effect of the leads on the field distribution.

In this paper we propose a new method, which eliminates connecting wires, for experimentally determining the interior field. The method consists of imbedding an isolated diode in the biological sample and measuring the local field by relying upon the unique reradiating characteristics of the diode. The basic thesis can be presented in the following form. Consider a diode placed in the field of a multifrequency electromagnetic source. The scattered energy from the diode would contain frequency components, other than those generated by the source, due to the inherent nonlinearity. The basic thesis is that the relative magnitudes of these components are uniquely related to the field intensity by the diode nonlinearity. Thus, by measuring amplitudes of the diode-generated frequency components, one can determine the field intensity. If the diode were now implanted in the biological specimen, the field at the implanting point would likewise be determined.

Manuscript received February 24, 1975; revised February 13, 1976 M. E. Brodwin is with the Department of Electrical Engineering, Northwestern University, Evanston, IL 60201.

A. Taflove is with the Division E, IIT Research Institute, Chicago, IL 60645 .

J. E. Matz is with Mark Products, Skokie, IL 60076.

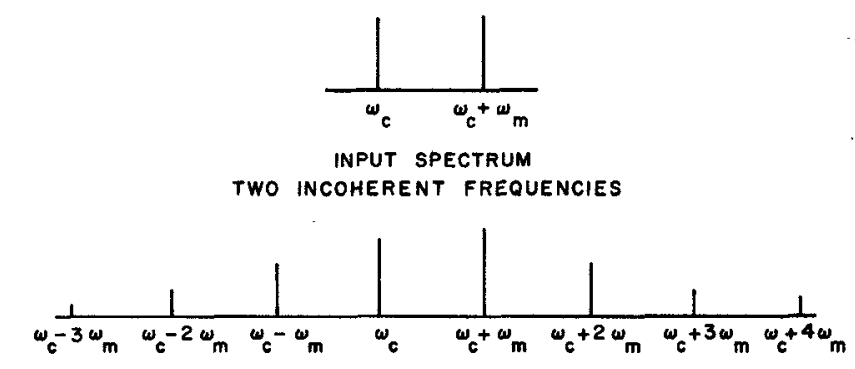

Fig. 1. Scattered spectrum-sidebands clustered about $\omega_{c}+\omega_{m} / 2$.

In this paper we shall first determine the theoretical amplitudes of the intermodulation products generated by a diode illuminated by a dual-frequency source, and then describe experiments in both waveguide and free space which support the basic thesis and provide values of the scattering parameters for the design of a suitable instrument.

\section{Theoretical ANALYsis}

The first part of this section establishes the scattered spectrum when the diode is illuminated by two sources with a small frequency difference. It will be shown that this spectrum contains intermodulation products whose amplitude ratios are unambiguously related to the intensity of the illuminating waves. The second part of this section derives a system equation which relates the observed scattered power to a simplified geometry and the characteristics of the diode.

The frequency of each component of the scattered field is most easily determined by inserting the expression for the incident-wave electric field into the power-series expansion of the $I-V$ characteristic of the diode [2]. We consider two sources, of frequency $\omega_{c}$ and $\omega_{c}+\omega_{m}$, which radiate equal-amplitude waves. At the diode, the incident electric field, given by

$$
E_{i}(t)=E_{0} \cos \omega_{c} t+E_{0} \cos \left(\omega_{c}+\omega_{m}\right) t
$$

is introduced into the power-series expansion for the diode

$$
I(t)=a_{1} E(t)+a_{2} E^{2}(t)+a_{3} E^{3}(t)+\cdots .
$$

After considerable manipulation, it may be shown that the diode current has frequency components symmetrically clustered about $0, \omega_{c}+\omega_{m} / 2,2 \omega_{c}+\omega_{m}, 3 \omega_{c}+3 \omega_{m} / 2$, etc. A typical spectral cluster, that centered about $\omega_{c}+$ $\omega_{m} / 2$, is shown in Fig. 1. This particular cluster is the most important one because its individual components result from the lowest order terms of the power series, and, 


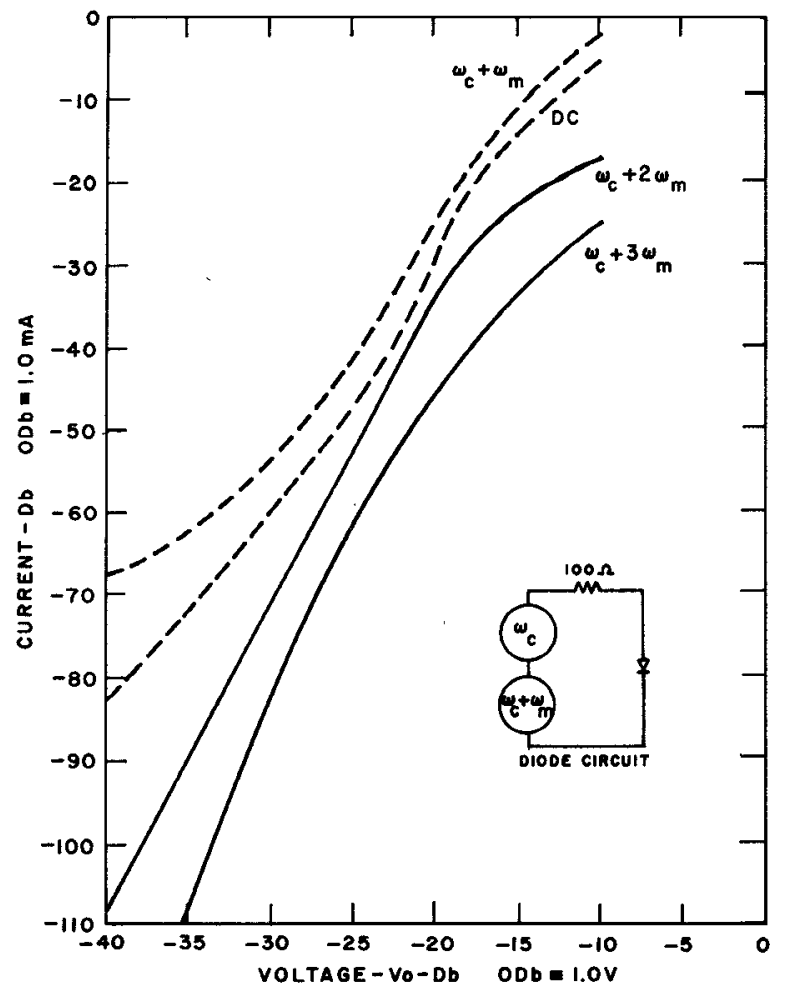

Fig. 2. Calculated values of the current at the intermodulation frequencies as a function of the amplitude of the sources.

therefore, are of greater amplitude than those of succeeding clusters.

Although the power-series representation is useful for predicting the frequency of each component of the scattered spectrum, it is of limited use in predicting the amplitude of the components. This limitation arises because a large number of terms is needed to describe accurately the diode characteristic for high-amplitude electric fields. A much more useful approach for estimating the power levels of the reradiated components is to use the diode equation. This equation is a more precise representation over a large range of power levels, and the important value, the reverse saturation current, is easy to obtain.

It is assumed that the problem of the diode scatterer may be modeled by the circuit shown in Fig. 2. The two sources represent the equivalent Thevenin generators of the diode when it is viewed as an antenna in lossy material. The resistance represents both the radiation resistance of the antenna and the residual series resistance of the diode. The simplifying assumptions are that the antenna impedance is not a function of the reradiated frequency and that there are no reactive components. The equation of the diode is

$$
I(t)=I_{0}\left(\varepsilon^{q V_{D}(t) / k T}-1\right)
$$

where

$$
V_{D}=V_{0} \sin \omega_{1} t+V_{0} \sin \omega_{2} t-R I(t) .
$$

A computer analysis yields the current as a function of time and then determines, via Fourier analysis, the frequency components of this current. In the computer simulation,

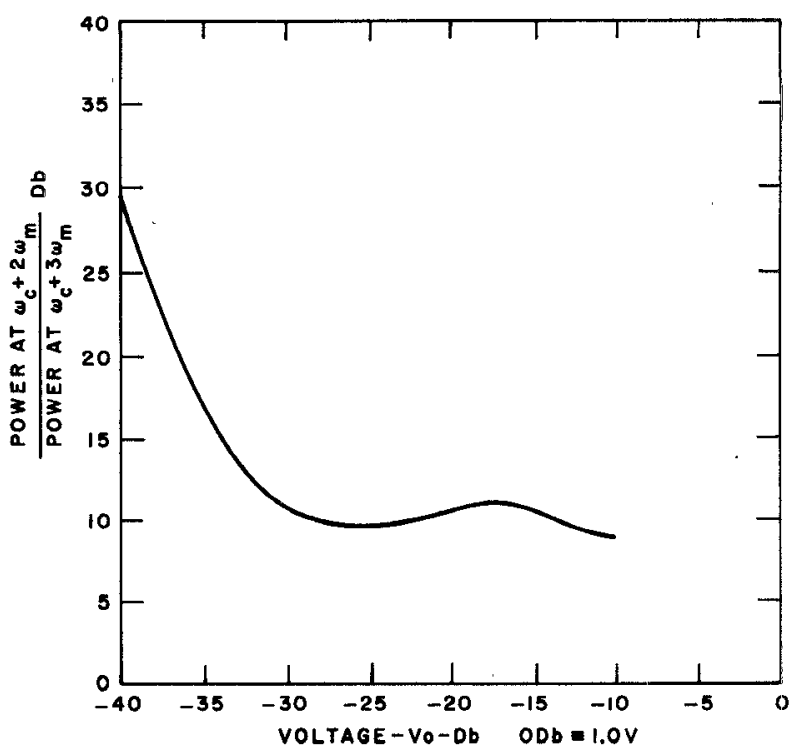

Fig. 3. Power ratio of the intermodulation frequencies as a function of the amplitude of the sources.

$f_{1}=4.0 \mathrm{~Hz}, f_{2}=5.0 \mathrm{~Hz}$, and (3) is solved by assuming a value for $I(t)$ and using Newton's method. The calculation is iterated for 10 steps and the result is $I(t)$. The Fourier series for $I(t)$ is then formed to determine the amplitude of the frequency components as a function of the voltage $V_{0}$. The resulting data appear in Fig. 2, which is a graph of the amplitudes of the components as a function of the amplitude of the applied voltage. At high levels of excitation, all curves tend toward a linear dependence upon the exciting level. As the level is decreased, the dc curve tends toward square law. The strongest intermodulation product, $\omega_{c}+2 \omega_{m}$, tends toward third order and the next important product, $\omega_{c}+3 \omega_{m}$, tends toward fifth order. These results are related to the power-series expansion in that the coefficients of the third- and fifth-order terms determine the amplitudes of the important intermodulation products at low levels.

The measurement of the interior fields is based upon the observation of the power ratio of the two strongest intermodulation products (Fig. 3). The most useful region is the low-level one in which there is a strong dependence of the observed ratio, $P_{\omega_{c}+2 \omega_{m}} / P_{\omega_{c}+3 \omega_{m}}$, upon the illuminating field. At high levels the ratio shows a distinct peak in the region of $-17 \mathrm{~dB}$, and as the field intensity decreases the ratio rises at a rapid rate. This unexpected peak has also been reported [3]. For observations at high levels, an ambiquity would be present which can be eliminated by varying the intensity of the illuminating field. If the field intensity were changed, the change in the ratio of the two intermodulation components would identify the region of the curve under observation. The high-level region shows a relatively flat dependence of the intermodulation ratio upon the field and would be ayoided since the accuracy in this region is poor.

Having established the basic principle of operation, we now proceed to derive an expression for the received power from a diode imbedded in a biological sample. The deriva- 




Fig. 4. Geometry for the calculation of received power.

tion is developed by means of the simplified geometry shown in Fig. 4. It is assumed that a source is located in a lossless medium at a distance $R_{1}$ from a planar interface. The scattering diode is imbedded in the lossy biological medium at a distance $R_{2}$ from the interface. The incident energy is presumed to arrive at normal incidence. It is assumed that the biological object is large compared to the wavelength and that the distances $R_{1}$ and $R_{2}$ are also large compared to the wavelength. The development resembles the derivation of the radar equation.

Assume that the total radiated power at the base frequencies is $P$ and is directed into an antenna of gain $G$. The incident power density at the interface is given by

$$
P_{i 12}=\frac{P G}{4 \pi R_{1}{ }^{2}}=\frac{E_{01}{ }^{2}}{2} g_{r 1}
$$

where $E_{01}$ is the amplitude of the incident wave at the interface and $g_{r 1}=1 / \eta_{1}$. On the right-hand side of the interface, the transmitted power density is

$$
P_{t 12}=\frac{E_{01}{ }^{2}}{2}\left|\tau_{12}\right|^{2} g_{r 2}
$$

where $\tau_{12}$ is the transmission coefficient for a source in region 1 , and $g_{r 2}=\operatorname{Re}\left(1 / \eta_{2}\right)$. Eliminating $E_{01}{ }^{2}$ from (4) and (5), we obtain a bridging relation between the power densities on both sides of the interface

$$
P_{t 12}=\frac{P_{i 12}\left|\tau_{12}\right|^{2} g_{r 2}}{g_{r 1}} .
$$

In terms of the power of the source

$$
P_{t 12}=\left(\frac{P G}{4 \pi R_{1}^{2}}\right)\left(\left|\tau_{12}\right|^{2} \frac{g_{r 2}}{g_{r 1}}\right) .
$$

The power density at the diode is affected by both the attenuation of the medium $\alpha$ and the inverse $R^{2}$ dependence

$$
P_{i d} \approx(P G)\left(\left|\tau_{12}\right|^{2} \frac{g_{r 2}}{g_{r 1}}\right) \frac{\varepsilon^{-2 \alpha R_{2}}}{4 \pi\left(R_{1}+\frac{R_{2}}{\sqrt{\varepsilon_{r}}}\right)^{2}} .
$$

The $\sqrt{\varepsilon_{r}}$ term arises from the bending of the rays at the interface. The wavefronts in medium 2 appear to originate from behind the actual source. Thus the intensity in medium 2 decreases more slowly than in medium 1 . The results of a more precise analysis [4], which includes losses, shows that for biological media, the lossless approximation is adequate.

A more exact analysis would also involve the solution of a boundary-value problem with a spherical wavefront at the interface. The inverse $R^{2}$ dependence of ( 8 ) should be viewed as a heuristic attempt to obtain a simple expression for first-order calculations. This viewpoint is further justified when the complicated nature of most biological materials is considered.

The scattering of the diode is expressed by a factor $\gamma_{n}$, which represents the ratio of the total isotropic scattered power at the intermodulation frequency to the incident power density at the base frequencies. The subscript $n$ corresponds to the chosen intermodulation frequency. The diode is now viewed as an isotropic source of strength, $\gamma_{n} P_{i d}$. The power density back at the interface is

$$
P_{i 21}=\frac{\gamma_{n} P_{i d} \varepsilon^{-2 \alpha R_{2}}}{4 \pi R_{2}^{2}}
$$

By means of the bridging relation (6), the transmitted power density on the left of the interface is

$$
P_{t 21}=\frac{P_{t 21}\left|\tau_{21}\right|^{2} g_{r 1}}{g_{r 2}} .
$$

Back at the antenna, the power density appears as

$$
\frac{\gamma_{n} P_{i d} \varepsilon^{-2 \alpha R_{2}}\left|\tau_{12}\right|^{2} g_{r 1}}{4 \pi \varepsilon_{r}\left(R_{1}+\frac{R_{2}}{\sqrt{\varepsilon_{r}}}\right)^{2} g_{r 2}} .
$$

The $\varepsilon_{r}$ term in the denominator accounts for the bending of the rays, now originating in the material, away from the normal at the interface. The apparent source is now closer to the interface.

By applying the concept of a receiving aperture, the relation between receiving aperture and gain, and inserting (8) the ratio of received power $P_{r, n}$ to the transmitted $P$, becomes

$$
\frac{P_{r, n}}{P} \approx \gamma_{n}\left|\tau_{12}\right|^{2}\left|\tau_{21}\right|^{2}\left(\frac{\varepsilon^{-4 \alpha R_{2}}}{(4 \pi)^{3} \varepsilon_{r}\left(R_{1}+\frac{R_{2}}{\sqrt{\varepsilon_{r}}}\right)^{4}}\right) \lambda^{2} G^{2} .
$$

In summary, the first term $\gamma_{n}$ represents the conversion efficiency; the ratio of the total isotropically radiated power at the scattered intermodulation frequencies due to the power density at the base frequencies. The next term accounts for the reflections at the interface. The term involving the distances describes both the inverse $R^{2}$ effect as well as the attenuation of the lossy medium, and the final term is the result of applying the concepts of gain and receiving aperture.

From (12) we also see that observation of a single intermodulation frequency is insufficient to determine the field intensity at the diode scatterer. With a single frequency observation, the field can be determined only if the trans- 
mission loss due to interface reflections and attenuation is known. In general, it is not possible to determine this loss for complicated structures. However, when two closely spaced intermodulation frequencies are observed, the transmission loss is nearly the same for both frequencies. In forming the observed power ratio

$$
\frac{P_{r, n}}{P_{r, n+1}}=\frac{\gamma_{n}}{\gamma_{n+1}}
$$

path loss factors common to both intermodulation products are cancelled. Therefore, (12a) is a function only of the electric field at the diode, the $I-V$ characteristic of the diode, and the loading effect of the tissue medium.

System calibration for a multitissue biological scatterer is accomplished in the following way. The diode is embedded deep within a phantom of one of the tissues. A simple shape is chosen so that the interior field is known. $P_{r, n} / P_{r, n+1}$ is measured as a function of the electric field at the diode. The procedure is repeated for another tissue of the biological scatterer. In this way, the effects of tissue loading upon the reradiation factor are determined empirically for each tissue of the scatterer.

If the diode were insulated [5], the response would be independent of the tissue parameters and the foregoing calibration procedure would not be necessary.

\section{Experimental Techniques and Results}

In this section we shall present the results of experiments with a diode in a waveguide instrumentation. The purpose of these initial experiments is to determine the relation between the reradiated power and the incident power density. One result of the waveguide experiment is an estimate of the value of the scattering parameter $\gamma$. A second series of experiments is described in which a low-power free-space instrument is assembled. This instrument is used to explore problems associated with the undesired generation of intermodulation products and to test the assumptions in the system equation (12).

\section{A. Waveguide Experiments}

The earliest experiments for observing the diode scattered signals used double-sideband amplitude modulation. The experiments were performed with a klystron source, operating $\mathrm{CW}$, with modulation imposed by a diode modulator in conjunction with a circulator. The energy, at $X$ band, was directed to a $1 \mathrm{~N} 23$ diode and the spectral content of the scattered signal was observed with a spectrum analyzer. The basic difficulty with this instrument was that the amplitude modulating method introduced its own spectral components which obscured those generated by the test diode.

At this point, it was decided to proceed with two separate sources and, in effect, to simulate a double-sideband suppressed-carrier incident signal. The apparatus is shown in Fig. 5. A klystron producing a signal at $f_{c}$ and a Gunn diode at $f_{c}+f_{m}$ are coupled via isolators and precision attenuators to a hybrid junction. The $E$ arm is used to sample the signals for frequency control and the difference fre-

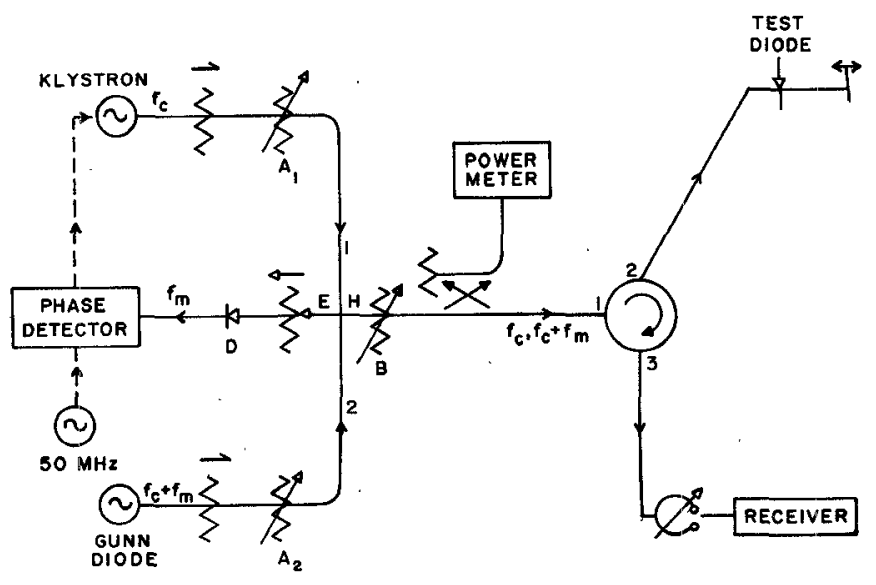

Fig. 5. Apparatus for the measurement of reradiated spectrum.

TABLE I

Schedule of Source ANd ObServed Frequencies

\begin{tabular}{lll}
\hline Kiystron & $\omega_{c}$ & $9600 \mathrm{MHz}$ \\
Gunn 0sc. & $\omega_{c}+\omega_{\mathrm{m}}$ & $9650 \mathrm{MHz}$ \\
$\mathrm{P}_{2}$ & $\omega_{c}+2 \omega_{\mathrm{m}}$ & $9700 \mathrm{MHz}$ \\
$\mathrm{P}_{3}$ & $\omega_{c}+3 \omega_{\mathrm{m}}$ & $9750 \mathrm{MHz}$ \\
$\mathrm{P}_{4}$ & $\omega_{c}+4 \omega_{\mathrm{m}}$ & $9800 \mathrm{MHz}$ \\
\hline
\end{tabular}

quency is produced by the diode $D$. The difference frequency, in the region of $50 \mathrm{MHz}$, is compared with a $50-\mathrm{MHz}$ reference oscillator in a phase-sensitive detector which generates a voltage to control the klystron. In this manner, the klystron frequency $f_{c}$ tracks the Gunn-diode frequency $f_{c}+f_{m}$ at a separation of $50 \mathrm{MHz}$. The $H$ arm of the hybrid junction is used to apply the two frequencies to the test diode. The combined amplitude is set by attenuator $B$, and individual powers as well as the combined power are monitored by the power meter. The two frequencies are directed through a circulator and into the test diode. The signals reradiated by the test diode proceed through the circulator and through a preselector into a calibrated receiver equipped with a precision IF attenuator. All data were taken with a fixed output of the receiver and relative values determined by the attenuator setting.

The goal of this preliminary experiment is to measure the reradiated intermodulation power, generated by the test diode, as a function of the total input power. The power output of each source was equalized by adjusting attenuators $A_{1}$ and $A_{2}$ as monitored by the power meter. The reference setting was a power level, into the circulator, of $2.0 \mathrm{~mW}$ corresponding to an attenuator $B$ setting of $10 \mathrm{~dB}$. An estimate of the incident radiation density is obtained by dividing the input power by the physical cross section of the waveguide, and was found to be $0.8 \mathrm{~mW} / \mathrm{cm}^{2}$. This level was chosen to be on the order of magnitude of maximum permissible radiation densities. The selected source and reradiated frequencies are shown in Table I. A correlating test was performed at a reradiated frequency of 9550 $\mathrm{MHz}, \omega_{c}-\omega_{m}$, to check the observation at $\omega_{c}+2 \omega_{m}$. It was found that the reradiated powers at these low fre- 


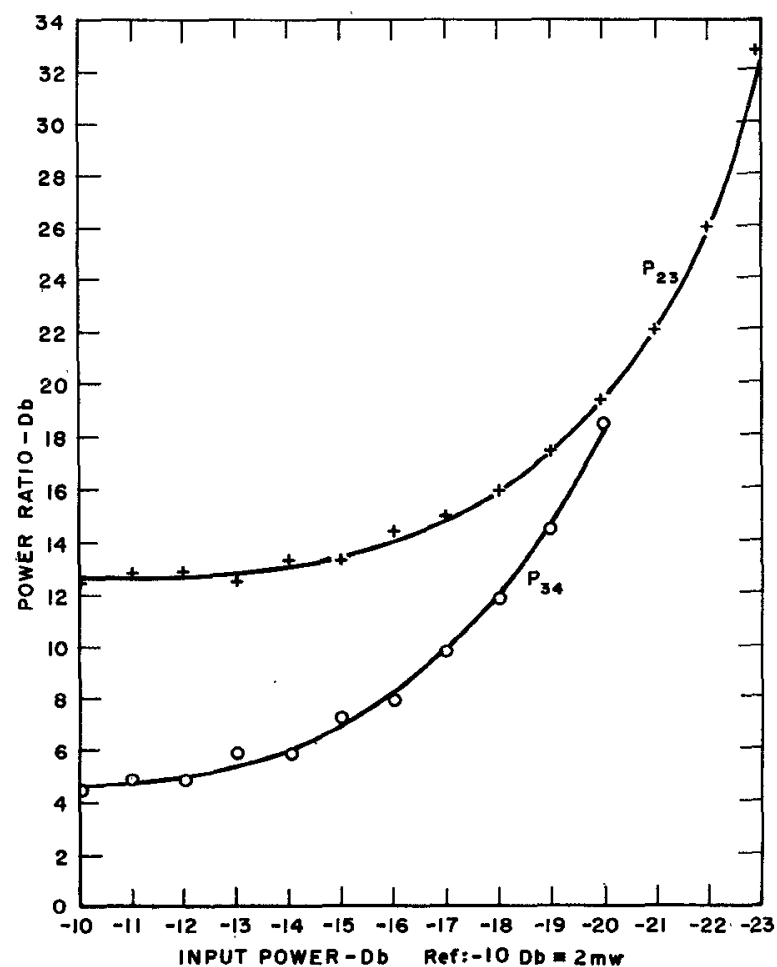

Fig. 6. Ratio of power at frequency $\left(\omega_{c}+n \omega_{m}\right)$ to the power at $\left(\omega_{c}+m \omega_{m}\right)$ versus input power.

quencies agreed quite well and thus the lower frequencies were ignored. A variety of resistive loads were placed upon the test diode, and it was found that the largest reradiated powers were observed with a short circuit. The principal data are the ratios of the powers at the intermodulation frequency $\omega_{c}+2 \omega_{m}$ to those at $\omega_{c}+3 \omega_{m}$, which are delineated as $P_{23}$ (Fig. 6). The abscissa represents the input power, with a reference level of $-10 \mathrm{~dB}$ corresponding to $2.0 \mathrm{~mW}$. At high powers, $P_{23}$ is relatively constant. In this region, the measurement of $P_{23}$ is a poor indicator of the power absorbed by the diode. However, as the power level is decreased, $P_{23}$ increases rapidly. In the region of -20 - to $-22-\mathrm{dB}$ input power, the slope of $P_{23}$ versus input power is very large. The inference is that a measurement of $P_{23}$ in this range would be a very accurate indication of the power incident upon the diode. The trend of the data follows the predicted variation of Fig. 3. The corresponding data for the next higher frequency ratio $P_{34}$ are also presented.

As the incident power increases, the observation would be shifted to higher order reradiated signals. The higher order ratios, $P_{67}$ and $P_{78}$, have a large slope at these power levels (Fig. 7). Thus it would be more accurate to use the higher order ratios for larger input power densities.

Having established the basic thesis that the ratio of reradiated powers is uniquely related to the power incident upon the diode, the next question is the practical one of designing a system suitable for this observation in biological samples. The essential data are the values of the isotropically

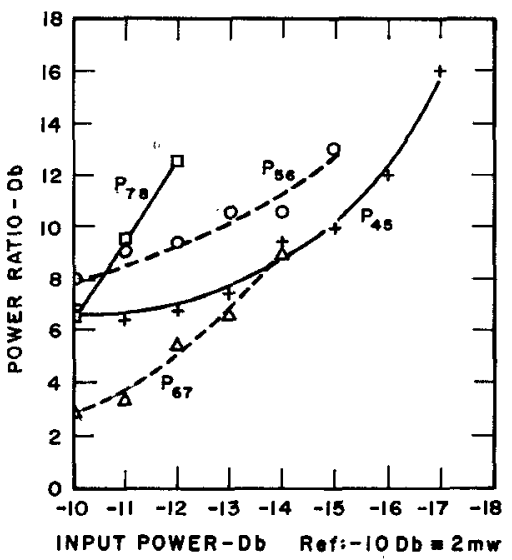

Fig. 7. Ratio of reradiated powers versus input power at the higher order frequencies.

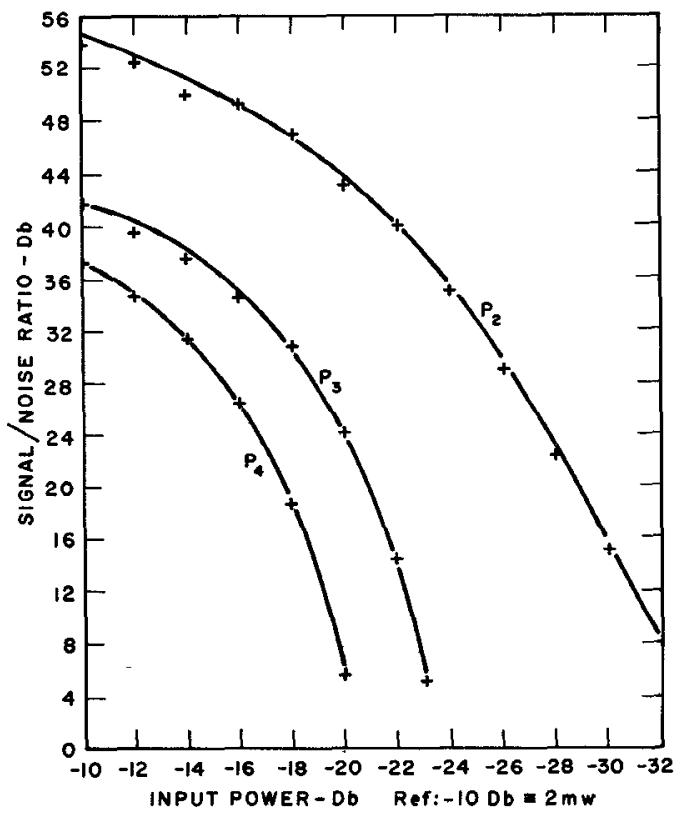

Fig. 8. $S / N$ ratio of the reradiated signal versus input power-noise level: $-83 \mathrm{dBm}$.

reradiated power normalized by the incident power density or $\gamma_{n},(12)$. The value for $\gamma_{n}$ can be inferred from the waveguide experiments. For example, to observe the power ratio $P_{23}$, it is sufficient to be able to observe $P_{3}$ since $P_{3}$ is the weaker of the two signals. From Fig. 8 it can be seen that there are two extreme values of $\gamma_{3}$. For example, if the incident power density is high, the total input power is $2.0 \mathrm{~mW}$, and the reradiated power is $42 \mathrm{~dB}$ above the receiver noise level of $-83 \mathrm{dBm}$. For this easily observed condition, the total reradiated power $P_{3}$ is roughly -40 $\mathrm{dBm}$ and the input power density is $2.0 \mathrm{~mW}$ divided by the physical cross section of the waveguide. In this case $\gamma_{3}$ is approximately $1 \times 10^{-8} \mathrm{~m}^{2}$. The other extreme value of $\gamma_{3}$ for this receiver occurs when $P_{3}$ is close to the noise level. Under these circumstances, the reradiated power is somewhat above the noise level, $-80 \mathrm{dBm}$, and the input power is approximately $23 \mathrm{~dB}$ below the reference level of 
$2.0 \mathrm{~mW}$ or $-10 \mathrm{dBm}$. Once again, dividing by the cross section of the waveguide, the smaller value of $\gamma_{3}$ is $2.33 \times$ $10^{-11} \mathrm{~m}^{2}$.

\section{B. Free-Space Experiments}

In order to determine the problems associated with an instrument which could be used to determine the interior fields of biological samples, a low-powered apparatus was assembled with a horn radiator and a highly sensitive receiver. A block diagram is shown in Fig. 9. With this instrument, the major problem associated with a measurement of the reradiated signal is the inadvertent generation of these signals within the instrumentation, and the unusual features appearing in Fig. 9 are inserted to solve this problem.

The two sources are delineated by $\omega_{c}$ and $\omega_{c}+\omega_{m}$. These are Gunn oscillators which were chosen for their increased power as compared with klystrons and their inherent frequency stability. Since they are basically diodes and will generate masking signals when injected with the other frequency, they must be very carefully isolated. For example, in the waveguide leading from $\omega_{c}$, a single isolator appears in the diagram. In the actual instrument, three cascaded ferrite isolators were used. The isolation is necessary to prevent any of the $\omega_{c}+\omega_{m}$ signal from entering $\omega_{c}$ and producing masking signals. A low-pass filter is inserted in this line to prevent harmonics of $\omega_{c}$, $2 \omega_{c}, 3 \omega_{c}, \cdots$, from being injected into $\omega_{c}+\omega_{m}$ and thereby producing masking signals arising from the mixing of harmonics. The $\omega_{c}+\omega_{m}$ frequency is locked onto the $\omega_{c}$ frequency by means of the $E$-arm branch of the hybrid junction. The two frequencies mix in a diode and produce a $25-\mathrm{MHz}$ difference frequency which is phase detected and used to control the frequency $\omega_{c}+\omega_{m}$. In this manner, the two frequencies are separated by $25 \mathrm{MHz}$ and the combination is free to drift as $\omega_{c}$ varies. To insure that the receiver follows this drifting combination, a fraction of the $\omega_{c}+\omega_{m}$ signal is extracted by means of a directional coupler and used as the local oscillator of the receiver. The receiver is a double-conversion system in which, a choice of second local oscillators is provided for selecting the 50 $\mathrm{MHz}, 2 \omega_{m}$ signal or the $75 \mathrm{MHz}, 3 \omega_{m}$ signal. The output of the receiver is in the audio range due to the natural differences of the $25-\mathrm{MHz}$ crystal-controlled oscillator in the phase detector and the crystal-controlled local oscillator in the receiver. The audio difference signal is the second IF frequency of the double-conversion receiver and the wave analyzer is used as a combination second IF and output indicator. The receiving system has an estimated sensitivity of $-136 \mathrm{dBm}$. To prevent masking signals from being generated in the mixer section of the receiver, a notch filter is placed at the receiver input to insure that the $\omega_{c}$ signal appearing at the output of the circulator is kept below the level of significant masking signal generation in the mixer diode. A tunable transmission cavity preselector is also inserted in this waveguide line to further suppress undesired signals.

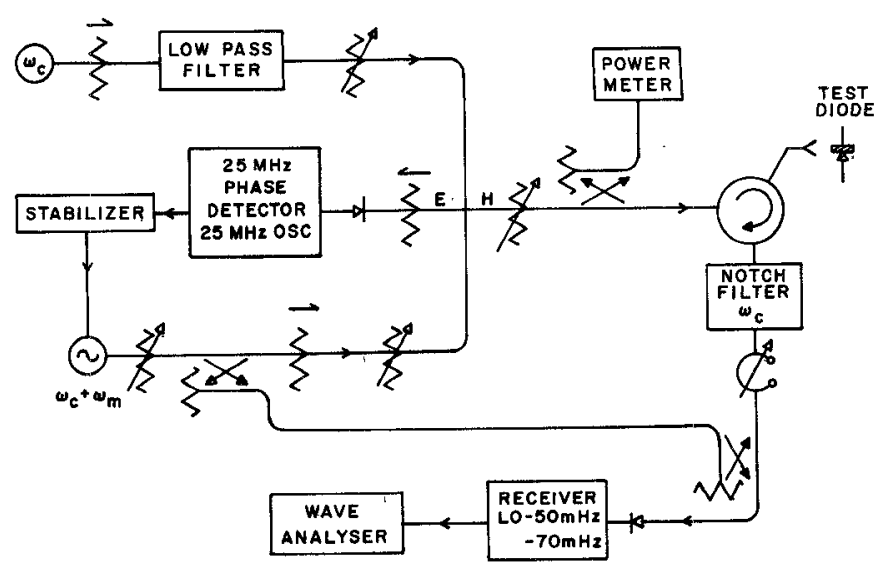

Fig. 9. Instrument for observing biological specimens.

In general, the sources of masking intermodulation products must be well isolated to prevent them from appearing at the receiver terminals. Thus the inherent diode construction of the Gunn oscillators is eliminated as a source of these signals by cascaded isolators in both Gunndiode lines. In a similar manner, isolators are provided ahead of the frequency-stabilizer mixer diode. To prevent extraneous mixing in the receiver mixer diode, filtering and preselection are used. To inhibit mixing at harmonics of the base frequencies, a low-pass filter has been employed in the $\omega_{c}$ branch. In this manner, only the desired reradiated signals from the test diode are observed at the output of the wave analyzer.

Experiments were performed to test the major elements of the theoretical analysis. In these experiments the apparatus of Fig. 9 was used to illuminate a IN23 diode in front of a standard gain horn. For the case of free-space illumination, (12) reduces to

$$
\frac{P_{r}}{P}=\frac{\gamma_{n}}{R^{4}}\left[\frac{G^{2} \lambda^{2}}{(4 \pi)^{3}}\right]
$$

Two experiments were carried out to test (13). In the first one, the apparatus was adjusted to observe the largest reradiated signal $P_{2}$. The diode was moved away from the horn until the received signal was at the noise level. The range for this test was $1 \mathrm{~m}$. A 12.0- $\mathrm{dB}$ horn was used and $\gamma_{2}$ was estimated from the waveguide experiments as $1.84 \times$ $10^{-10} \mathrm{~m}^{2}$. The calculated value from (13) of the transmitted power was roughly $1 \mathrm{~mW}$. This value is within an order of magnitude of the actual transmitted power, $10 \mathrm{~mW}$. The diode was then moved closer to the horn and the apparatus set for $P_{3}$. This power was just observed when the diode was about $15 \mathrm{~cm}$ from the horn. The calculation was repeated with a value of $\gamma$ equal to $2.33 \times 10^{-11} \mathrm{~m}^{2}$. The calculated transmitted power was 1-mW and again the result represents an order of magnitude confirmation of the theory. Other experiments were performed to determine the optimum loading of the diode: the bare crystal cartridge, a shortcircuiting loop antenna and a short dipole antenna. The preliminary indications are that the largest reradiated signal 
was observed for the basic diode without any antenna structure. In this manner, the problems associated with the instrument have been explored and it is now possible to consider future, more useful instruments for observing the interior field of biological specimens.

\section{Application to Biological Materials}

In this section we shall apply the system equation to the problem of determining the interior fields. The results are graphs of implantation depth versus the power required of the source. To obtain these graphs each term in (12) is appropriately evaluated. The scattering parameter is inferred from the previous experimental data, and the biological terms are evaluated by assuming that the biological material can be represented by loss tangents varying between 0.1 and 0.5 . The spacing between the antenna and the sample is based upon the far-field criterion.

The first term in the system equation to be considered is the scattering parameter $\gamma$. The results of the previous waveguide experiments showed that the value of $\gamma$ is a strong function of the incident power density, as it must be in order for the system to function. To estimate how the instrument would function with biological samples, we choose a reasonably small value of $\gamma$. The lowest observable value in the waveguide experiments was $2.33 \times 10^{-11} \mathrm{~m}^{2}$. This value corresponds to an input power of $-10 \mathrm{dBm}$ and was calculated with a receiver sensitivity of $-83 \mathrm{dBm}$. In the free-space instrument, previously described, the minimum detectable signal was estimated at $-136 \mathrm{dBm}$. Thus a chosen value of $\gamma=2 \times 10^{-11} \mathrm{~m}^{2}$ is significantly greater than the minimum detectable $\gamma$ of the free-space instrument. This choice introduces a safety margin to allow for significant differences between the $\gamma$ of an unbounded system and the measured $\gamma$ of the waveguide experiment. The next term to be estimated is the transmission parameter, $\left|\tau_{12}\right|^{2}\left|\tau_{21}\right|^{2}$. The transmission term is related to the intrinsic impedances by

$$
\left|\tau_{12}\right|^{2}\left|\tau_{21}\right|^{2}=\eta_{1}{ }^{2}\left|\eta_{2}\right|^{2}\left[\frac{4}{\eta_{1}{ }^{2}+2 \eta_{2 r} \eta_{1}+\left|\eta_{2}\right|^{2}}\right]^{2}
$$

where $\eta_{1}$ is the intrinsic impedance of the lossless medium, and $\eta_{2}$ is the intrinsic impedance of the lossy medium. $\eta_{2}$ is presumed to be complex and can be expressed in terms of a complex dielectric or a conductivity formulation. $\eta_{2 r}$ is the real part of the complex intrinsic impedance $\eta_{2}$. To obtain an estimate of the reflection effects, we assume that the biological sample can be represented by a dielectric constant, $\varepsilon_{r}=80$, and by loss tangents varying between 0.1 and 0.5 . A calculation of the transmission parameter yields values between 0.121 and 0.131 when using the exact expression, (14). Since $\varepsilon_{r}$ is large, the error, introduced by neglecting the complex nature of the medium, is small. A calculation, based solely upon the real part

$$
\left|\tau_{12}\right|^{2}\left|\tau_{21}\right|^{2}=\frac{16 \varepsilon_{r}}{\left(\sqrt{\varepsilon_{r}}+1\right)^{4}}
$$

yields a result, 0.126 . Consequently, the lossy nature of the
TABLE II

Assumed Values for Required Power Calculation

\begin{tabular}{lll}
\hline & $910 \mathrm{MHz}$ & $2,450 \mathrm{MHz}$ \\
\hline$\gamma$ & $=2.33 \times 10^{-11} \mathrm{~m}^{2}$ & $2.33 \times 10^{-11} \mathrm{~m}^{2}$ \\
$G$ & $10.0 \mathrm{~dB}$ & $0.0 \mathrm{~dB}$ \\
$\left|\tau_{12}\right|^{2}\left|\tau_{21}\right|^{2}=0.13$ & 0.13 \\
for $\tan \delta=0.1 \quad \alpha=8.5$ nepers $/ \mathrm{m}$ & $\alpha=23.8 \mathrm{nepers} / \mathrm{m}$ \\
for $\tan \delta=0.5 \quad \alpha=41.4$ nepers $/ \mathrm{m}$ & $\alpha=116 \mathrm{nepers} / \mathrm{m}$ \\
$\mathrm{R}_{1}$ & $=1.34 \mathrm{~m}$ & $0.5 \mathrm{~m}$ \\
$\mathrm{P}_{\mathrm{r}}$ & $=-136 \mathrm{dBm}$ & $-136 \mathrm{dBm}$ \\
\hline
\end{tabular}

medium need not be considered when calculating the transmission parameter.

The calculation of the attenuation constant follows the well-known expression [6]

$$
\alpha=\omega \sqrt{\frac{\mu \varepsilon^{\prime}}{2}\left[\sqrt{1+\left(\frac{\varepsilon^{\prime \prime}}{\varepsilon^{\prime}}\right)^{2}}-1\right]} .
$$

The distance $R_{1}$ from the antenna to the inferface is chosen as the shortest distance which fulfills the criterion for the far field of the antenna

$$
R_{1} \geq \frac{2 D^{2}}{\lambda}
$$

where $D$ is the major dimension of the antenna aperture. This range can be expressed in terms of gain and wavelength in the following manner. Assume that the physical aperture is twice the receiving aperture. The physical receiving aperture $A_{r p}$ is then

$$
A_{r p}=\frac{G \lambda^{2}}{2 \pi}
$$

Assuming a circular aperture, one can solve for the diameter as a function of $G$ and $\lambda$

$$
D=\frac{\lambda}{\pi} \sqrt{2 G}
$$

In terms of the desired parameters, the minimum range is given by

$$
R_{1}=\frac{4 G \lambda}{\pi^{2}} .
$$

The most useful way of viewing the system equation (12) is to consider the implantation depth $R_{2}$ as the independent variable and solve for the power of the source. The schedule of values for this calculation is presented in Table II.

A graph of the implantation depth versus the source power is shown in Fig. 10. An example of the use of this graph is to consider an experiment for the determination of the field in fat, $\tan \delta=0.1$. At $910 \mathrm{MHz}$, the field distribution in $20 \mathrm{~cm}$ of fat could be determined without exceeding an illuminating density of $10 \mathrm{~mW} / \mathrm{cm}^{2}$. For 


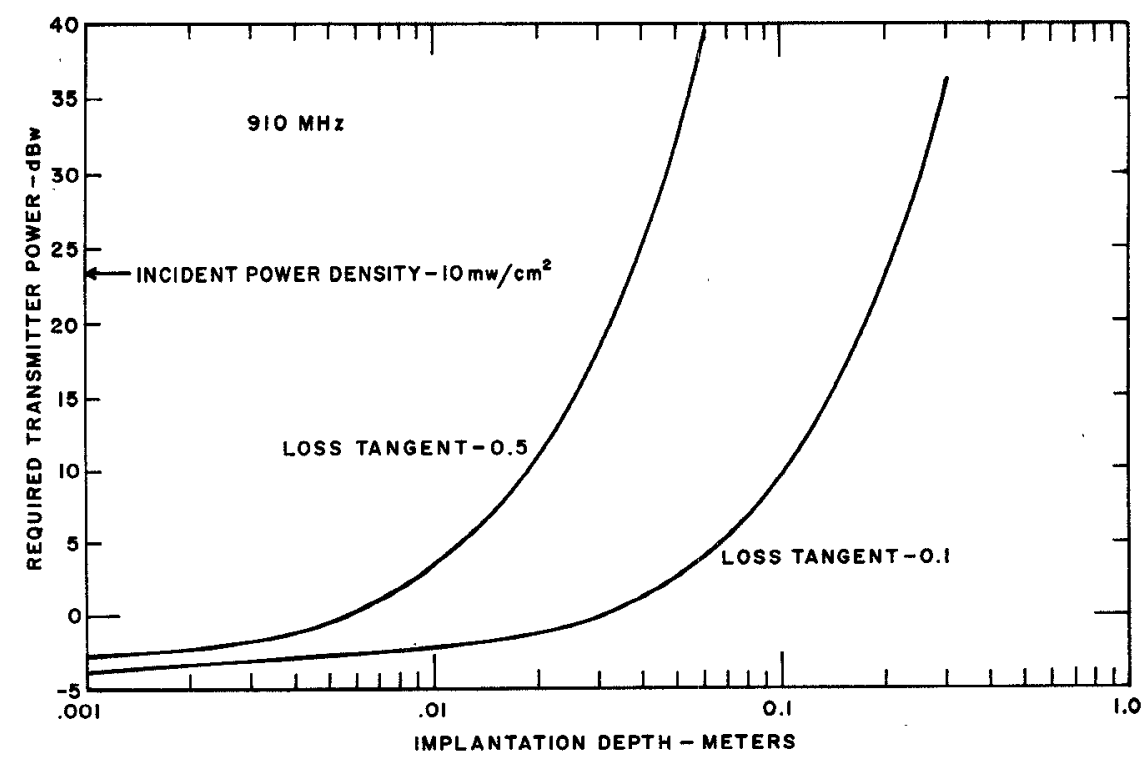

Fig. 10. Required transmitter power versus implantation depth-910 $\mathrm{MHz}$.



Fig. 11. Required transmitter power versus implantation depth-2450 MHz.

tissues with a high water content $(\tan \delta=0.5)$, such as the eye, the diode could be implanted up to a distance of $4 \mathrm{~cm}$ and the field distribution in the eye could be determined. The corresponding information for experiments at 2450 $\mathrm{MHz}$ are shown in Fig. 11. In this manner, an instrumentation can be designed for almost any study of the field distribution in biological materials at suitable frequencies.

\section{ACKNOWLEDGMENT}

The authors wish to thank S. Kahn and H. Kwok for their many helpful discussions and assistance with the experimental portions of this work.

\section{REFERENCES}

[1] For a recent review of this problem, see: S. M. Michaelson, "Review of a program to assess the effects on man from exposure to microwaves," The Journal of Microwave Power, vol. 9, no. 2, pp. 147-161, June 1974.

[2] M. E. Brodwin, C. Johnson, and W. Water, "Low level synchronous mixing," Proc. IRE, vol. 41, p. 969, August 1953.

[3] R. L. Stengel, "Nonlinear scattering dipole for electromagnetic field measurements in plasmas," Rev. Sci. Inst., vol. 45, no. 5 , p. 626, May 1974.

[4] J. A. Stratton, Electromagnetic Theory. New York: McGraw-Hill, 1941.

[5] R. W. P. King, "The many faces of the insulated antenna," Proc. IEEE, vol. 64, pp. 228-238, Feb. 1976.

[6] S. Ramo, J. R. Whinnery, and T. Van Duzer, Fields and Waves in Communication Electronics. New York: John Wiley and Sons, 1965. 